\title{
DIE ERSTELLUNG EINES LERNMEDIUMS MIT DER CAMTASIA STUDIO 8 SOFTWARE ZUM THEMA „ALLTAG” AUS DEM BUCH STUDIO D B1
}

\author{
Meylin Fransiska Panjaitan \\ A. Sahat Pardamean \\ Herlina JP Harahap
}

\begin{abstract}
AUSZUG
Das ZieldieserUntersuchung is es, um Lernmediumsmit der Camtasia Studio 8 Software zum Thema „Alltag" aus dem Buch Studio d B1 zuerstellen. In dieserUntersuchungwirdErstellungmethodeangewendet. Der Prozess der Erstellung der Lernmediumsmit der Camtasia Studio 8 Software zum Thema „Alltag” aus dem Buch Studio d B1 bestehtaus der Erklärung der Phasen von Theorie Ricky und Klein. Das Ergebnis der ErstellungeinesLernmediumsmit der Camtasia Studio 8 Software zum Thema „Alltag” aus dem Buch Studio d B1 sieht so aus.Das erstellteLernmediumbesteht aus 5 Videos.Dieses erstellteLernmediumkönntenbeimLernprozessan der Universitätbenutzen.
\end{abstract}

Schlïsselwörter: Lernmedium, Alltag

\section{EINLEITUNG}

\section{A. Der Hintergrund}

Das Lernmedium ist ein wichtiger Bestandteil des Lernprozesses. Das Lernmedium ist sehr einflussreich auf die Ergebnisse des Unterrichts, weil das Lernmedium die Lerneigenschaft steigern kann, wie Kompetenzen erreichen. Das Lernmedium kann effektiver und effizienter Lernprozesserzeugen.

Es gibt 4 Kompetenzen im Deutschunterricht, nämlich Hörverstehen, Sprechfertigkeit, Leseverstehen und Schreibfertigkeit. Die vier Kompetenzen gruppiert im Buch Studio d B1. Im 4. Semester werden das Buch Studio d B1 von den Studenten benutzt. Im Studio d B1 gibt es viele Themen. Ein von vielen Themen im Studio d B1 ist Alltag. Das Thema „Alltag” im Buch Studio d B1 enthält auf der Seite 28. Alltag ist ein interessantes Thema zu lernen, weil es über die Aktivitäten erzählt, wie in der Bibliothek, auf der Bank, Stress im Alltag und Lachen ist gesund.

Im Lernprozess wird neues und innovatives Lernmedium sehr wichtig benutzt, um die Lernziele und Kompetenzen zu steigern, weil das Lernmedium sehr einflussreich auf der Aufmerksamkeit der Studenten ist. Anthony (1936:96) stellt fest, dass das 
Medium eine besondere Strategie ist (vgl. Arsyad, 2013:5). Kemp und Dayton (vgl. Arsyad, 2002:15) stellen fest, dass die Verwendung das Medium in dem Lernprozess ein Wunsch und ein neues Interesse aufwecken konnen und aufweckt die Motivation und die Stimulanz der Lernaktivitäten und bringt sogar der Psychologischeinfluss für die Studenten.

Von 05 bis 08 April 2016 wurde den Studenten in der Deutschabteilung die Frage gegeben. Beim Lernen über Alltag, haben die Studenten Langweile. Basierend auf dieser Beobachtung lernen die Studenten über Alltag nur mit dem Buch Studio d B1 und hören nur von dem CD, sodass die Studenten interessieren sich nicht zu lernen.

Um das Problem zu lösen wird etwas Kreativität durch eine Entwicklungsmedium gebraucht, sodass der Deutschunterricht effizienter und effektiver sein wird. Eine Erstellung des Lernmediums ist Camtasia Studio 8. Camtasia Studio 8 ist eine Software, um ein Video auf zu nehmen. Diese Software kann verwendet werden, um eine Medien-based Learning, multimedia und e-Learning zu erstellen.

Camtasia Studio hat die Fähigkeit, Geräusche aufnehmen, die auf dem Bildschirm vorhanden sind, einschließlich Desktop Aktivitäten, Powerpoint-Präsentationen, gesprochenen und Webcam video.Das Camtasia Medium zum Thema "Alltag” wird noch nicht benutzt. Camtasia Studio 8 hat die Vorteile, dass sie Lernmaterial in verschiedenen Formen zeigen können, wie Beispieleweise in einschließlich Desktop Aktivitäten, Powerpoint-Präsentationen und Webcam Videoform.Indriana (2011) sagt aus, diese Software kann verwendet werden, um ein Lernmedium, Multimedia und eLearning zu erstellen. Daher wird mit dem Camtasia studio 8 Medium, um die Sprechfertigkeit und Hörverstehen Kompetenzen der Studenten zu steigern erwartet können.

Basierend auf der obigen Erklärungen interessiert sich die Verfasserin der Untersuchung für „Die Erstellung Eines Lernmediums mit der Camtasia Studio 8 Software zum Thema ,Alltag” aus dem Buch Studio D B1”.

\section{THEORETISCHE UND KONZEPTUELLE GRUNDLAGEN}

Der Begriff des Entwicklungsmodell 
Sugiyono (2015:297) erklärt, dass die bestimmte Produkte produzieren um die Wirksamkeit des Produkts zu testen, damit es, in der Gesellschaft funktionieren kann, dann die Forschung notwendig ist, um diese Produkte zu testen.

Richey und Klein (2009) stellen fest: das Design und die Forschung ist eine systematische Untersuchung, um einem Produktsdesign $\mathrm{zu}$ machen, die das Design/Produkt zu entwickeln und bewerten die Leistung diesem Produkt, mit dem Ziel der empirischen Daten, die das Design und die Forschung können als Grundlage für die Herstellung der Produkte und Modelle, die in Lern verwendet werden.

Borg und Galle (1998) stellen fest, dass was Forschung und Entwicklung ist? Forschung und Entwicklung ist der Prozess/Methode zum prüfen und entwickeln Produkte verwendet. (vgl. Sugiyono, 2015:28)

Die andere Quellen stellt fest, dass Forschung und Entwicklung ist eine systematische Weise die eine Design entwickelt verwendet und entwickelt ein Programm und Produk, die die Kriterien erfüllen (www.eric.ed.go) können (vgl. Sugiyono 2015:29).

Basierend auf dem Verständnis von Forschung und Entwicklung, stellen sich folgenden, dass jede Entwicklung ein Produkt produzieren würde, durch den Prozess der Planung, das Produk und die Bewertungsgültig produziert worden.

Von den zuvor genannten Entwicklungsmodellen wird in dieser Untersuchung das Richey und Klein gewählt. Das Richey und Klein ist einfach und systematisch in der Verwendung. Es besteht aus drei Schritte:

1. Die Planung (das Design)

Das Design ist ein Produktplan für einen bestimmten Zweck zu erstellt werden. Die Planung beginnt mit einer Bedarfsanalyse durch Forschung und Studium der Literaturdurchgeführt.

2. Die Produktion

Die Produktion ist die Tätigkeit der Herstellung von Produkten basierend auf dem Entwurf, der erstellt wurde.

3. Die Bewertung (Evaluation)

e Evaluation ist eine Aktivität zu prüfen, wie das höchstleitungsprodukt um die Spezifikationen zu erfüllt wurden.

\section{Camtasia Studio 8}


Camtasia ist von TechSmith Coorporation entwickelte Software (Software). Camtasia selbst wird verwendet, um alle Aktivitäten auf dem Bildschirm aufzeichnen. Diese Software kann verwendet werden, um eine Medien-based Learning, multimedia und e-Learning zu erstellen.

Camtasia Studio hat die Fähigkeit, Geräusche aufnehmen, die auf dem Bildschirm vorhanden sind, einschließlich Desktop Aktivitäten, Powerpoint-Präsentationen, gesprochenen und Webcam video. Camtasia Studio ist eine Komplettlösung für die Erstellung und Aktivität der Propesional video-Desktop PC schnell. Jeder kann zeichnen und erstellen ein full-Motion video-Lektion oder eine Präsentation.

Camtasia Studio besitzt die Fähigkeit, video Aufzeichnung der Ergebnisse (Bild) in 3 Arten von Dateien von Format und Direndering, nämlich zu speichern:

Camtasia Studio 8 bestandteile der Studie besteht aus vier Hauptkomponenten in Abhängigkeit von der Anwendung. Wie bei den vier Komponenten sind:

1. Sie können den Bildschirm nehmen.

2. Die Aufzeichnung gesprochener.

3. Die Aufnahme PowerPoint

4. Das Medium importieren.

Die Materie

Das Thema "Alltag"

Das Thema der Alltag wird im vierten Semester gegeben und umfasst mehrere Unterthemen. Im Deutschunterricht im vierten Semester, geht es um Zeitpunkte, Alltag, Generation, usw. Aber Alltag ist eine interssante Themen zu lernen, weil es über die Aktivitäten erzählt, wie Stress im Alltag, auf der Bank, Lachen ist gesund und in der Bibliothek. Im Thema Alltag wird auch die Grammatik lernen:

(Konjunktiv II (Präsens) der Modalverben sollte; müsste; könnte), (Konjunktionen mit darum, deshalb, deswegen), (Imperativ), (graduierende Adverbien: sehr, besonders, ziemlich).

\section{UNTERSUCHUNGSMETHODOLOGIE}

Die Daten und die Datenquelle

Die Daten in dieser Untersuchung sind Bilder und Wörter aus dem Buch Studio d B1 und einige Videos aus Youtube. Diese Bilder (von Internet, die Quelle: 
www.google.co.id), Wörter (von Studio d B1 seite 30, seite35, seite 37, seite 38, seite 39 und seite 245), www.landasanteori.com, Herbst 2014), und Video (Herbst 2014) werden in Videos auf zu nehmen.

\section{Die Skizze der Untersuchung}

Diese Untersuchung benutzt die Theorie von Richey und Klein. Diese Theorie wird schon im Kapitel II erklärt. In dieser Theorie besteht aus drei Schritte. Die Phase der Erstellung sind:

1. Die Planung (Design)

2. Die Produktion

3. Die Bewertung und Verbesserung (Evaluation)

\section{ERGEBNIS DER UNTERSUCHUNG}

\section{Der Prozess der Erstellung}

\section{Die Planung (Design)}

Die „Planung” ist die erste Phase. Bei der Planung wurde die Umfrage durchgeführt. Das Ergebnis (von) der Umfrage ist, dass die Studenten beim Lernen über Alltag, Langeweile haben. Das zeigt, dass die Studenten sich nicht am Lernen interessieren. Das Thema „Alltag” wird von Studenten im 4.Semester gelernt. Die Datenquelle dieses Themas sind Studio d B1 und Internet. Nachdem alle Materialien gesammelt wurden, werden sie geordnet und zusammengefasst.

\section{Die Produktion}

Basierend auf der ersten Phase wird das Lernmedium erstellt. Der Zweite Schritt in dieser Untersuchung ist die Produktion.

\section{Die Bewertung und Verbesserung (Evaluation)}

Das ist die letzte Phase, um ein interessantes Lernmedium zu erstellen. In dieser Phase wurde das Video schon erstellt. Dann werden die Prüfung und die Validierung durchgeführt. So kann herausgefunden werden, ob das Video schon gut genug ist oder noch verbessert werden sollte.

Dieses Video wird von Experten geprüft. Die Experten sind eine Dozentin und ein deutscher Muttersprachler. Die Experten haben einen pädagogischen Hintergrund.

\section{SCHLUSSFOLGERUNG}

Nach den Untersuchungsergebnissen fallen die Schlussfolgerungen folgendermaßen aus: 
1. Der Prozess der Erstellung eines Lernmediums mit der Camtasia Studio 8 Software zumThema „Alltag” aus dem Buch Studio d B1 besteht aus der Erklärung der Phasen von Richey und Klein. Diese sind: (i) Die Planung, (ii) Die Produktion, (iii) die Bewertung und Verbesserung (Evaluation).

2. Die Ergebnisse der Erstellung eines Lernmediums mit der Camtasia Studio 8 Software zumThema „Alltag” sind:

a. Diese Untersuchung hat die Videos erstellt.

b. Das Video Lernmedium über „Alltag” wurde von Experten evaluiert.Das Design wurde von einem Designexperten evaluiert. Danach wird die Evaluation der Wörter und der Sprache in dem Video korrigiert, was von dem Muttersprachler gemacht wird.

\section{LITERATURVERZEICHNIS}

Anthony N, Robert; Gonvindarajan Vijay; Management Control System; McGra-Hill, International Edition.

Ariani, Niken dan Dani Haryanto. 2010. Pembelajaran Multimedia di Sekolah. Jakarta: Prestasi Pustaka.

Aripin. 2009. Step by step membuat video tutorial menggunakan Camtasia Studio. Bandung: Oase Media.

Ariwibowo Syamsul dan Rudjiono Daniel. 2014. Perancangan Media Pembelajaran Interaktif Bahasa Arab Kelas VII Madrasah Tsanawiyah Al Uswah Bergas. http://Jurnalonline.um.ac.id/.../artikel/artikel552FE9AF59C4346253B49958 6924C43. pdf, gelesen am 08 April 2017.

Arsyad, Azhar. 2015. Media Pembelajaran. (Cetakan ke-16). Jakarta: PT Raja Grafindo Persada.

Borg R Walter; Gall D. Meredith. 2003, Educational Research, Longman, New York. Camtasia studio 7 the essentials. Iconlogic. Inc 2010.

Daryanto, 2009, Panduan Proses Pembelajaran. Teori dan Praktik Dalam Pengembangan Profesionalisme Guru, Jakarta: AV Publisher.

Fathurohman, Pupuh dkk. 2009. Strategi Belajar Mengajar. Bandung: PT.Rafika Aditama. 
https://jurnal.unimed.ac.id/2012/index.php/studia/index

Flash, Babang. 2016. Pengenalan Tools dan Fitur Bagi Pemula - Youtube https://www.youtube.com/watch?v=mH1mdGhjqls gesehen am 08. April 2017.

Flash, Babang. 2016. Cara Mudah Buat Efek Transisi Animasi -

Youtubehttps://www.youtube.com/watch?v=OLbFBfeoJe0gesehen am 08. April 2017.

Fundamental of camtasia studio, TechSmith Corporation, 2010.

Gagne, R.M (Ed.) 1987. Instructional Technology: Foundations. Hillsdale: Lawrance Erlmaum Associates, Publishers.

Gerlach, V.G. dan Ely, D.P. 1971. Teaching and Media. A Systematic Approach. Englewood Cliffs: Prentice Hall,Inc.

Get great audio Camtasia studio, TechSmith Corporation, 2010.

Hermann Funk. 2013. Studio d B1. Jakarta: Katalis.

Indriana. Diana. 2011. Ragam Alat Bantu Media Pengajaran. Yogyakarta: Diva Press.

Kemp, J.E. dan Dayton, D.K. 1985. Planning and Producing Instructional Media (Fifth Edition). New York: Harper \& Row, Publisher.

Latuheru, J.D. 1993. Media Pembelajaran dalam Proses Belajar Mengajar Kini. Ujung Pandang: Penerbit IKIP Ujung Pandang.

Nuary, Fandy., Havid A., 2014. Using Camtasia Studio 8 To Produce Learning Video To Teach English Through E-Laerning. International Journal. FBS State University of Padang.

http://ejournal.unp.ac.id/index.php/jelt/article/viewFile/4384/3442,pdf, gelesen am 08 . April 2017.

Prasetyo Adi Arista, 2014. Menjadi Pembuat Film Andal dengan Camtasia Studio 8 : PT Elex Media Computer.

Richey C, Rita; Klein D James, 2009. Design and Development Research. Routledge, New York, London. 
Studia: Journal des Deutschsprogramms p-ISSN 2301-6108 e-ISSN 2654-9573

https://jurnal.unimed.ac.id/2012/index.php/studia/index

Seels, B.B. dan Richey, R.C.1994. Instructional Technology: The Definition and Domain of the Field. Washington, DC.: Association for Educational Communicationand Technology.

Sugiyono, 2015. Metode Penelitian \& Pengembangan. Bandung: Alfabeta.

Techsmith. 2005. Camtasia Studio Show The World. Amerika.

Widiarto, Sony. 2011. Membuat Video Tutorial dengan Camtasia Studio 7. staff.unila.ac.id. pdf. Gelesen am 08. April 2017.

Meylin Fransiska Panjaitan is an alumni of German language study program of Unimed, Medan 
Studia: Journal des Deutschsprogramms p-ISSN 2301-6108 e-ISSN 2654-9573

https://jurnal.unimed.ac.id/2012/index.php/studia/index 